PROCEEDINGS OF THE

AMERICAN MATHEMATICAL SOCIETY

Volume 126, Number 2, February 1998, Pages 461-470

$\mathrm{S} 0002-9939(98) 04151-3$

\title{
SCHUR-CONVEX FUNCTIONS AND ISOPERIMETRIC INEQUALITIES
}

\author{
XIN-MIN ZHANG
}

(Communicated by J. Marshall Ash)

\begin{abstract}
In this paper, we establish some analytic inequalities for Schurconvex functions that are made of solutions of a second order nonlinear differential equation. We apply these analytic inequalities to obtain some geometric inequalities.
\end{abstract}

\section{INTRODUCTION}

The Schur-convex function was introduced by I. Schur in 1923 [14] and has many important applications in analytic inequalities. The following definitions and examples can be found in many references such as [3], [9], and [15].

Definition 1.1. An $n \times n$ matrix $S=\left[s_{i j}\right]$ is called a doubly stochastic matrix if $s_{i j} \geq 0$ for $1 \leq i, j \leq n$, and

$$
\sum_{j=1}^{n} s_{i j}=1, \quad i=1,2, \cdots, n ; \quad \sum_{i=1}^{n} s_{i j}=1, \quad j=1,2, \cdots, n .
$$

Examples 1.2. (a) A permutation matrix is a doubly stochastic matrix. (A permutation matrix is a matrix obtained by permuting the rows of the identity matrix.)

(b) $S=\left[s_{i j}\right]$ with $s_{i j}=\frac{1}{n}, 1 \leq i, j \leq n$, is a doubly stochastic matrix.

Let $I^{n}=I \times I \times \cdots \times I$ ( $n$ copies $)$, where $I$ is an interval of the real number line $\mathbf{R}$. We are interested in the following special multivariable functions.

Definition 1.3. $f: I^{n} \longrightarrow \mathbf{R}(n>1)$ is called Schur-convex if for every doubly stochastic matrix $S$,

$$
f(S \mathbf{x}) \leq f(\mathbf{x})
$$

for all $\mathbf{x} \in I^{n}$. It is called strictly Schur-convex if the inequality is strict; $f$ is called Schur-concave (resp. strictly Schur-concave) if the inequality (1) is reversed.

Definition 1.4. $f: I^{n} \longrightarrow \mathbf{R}(n>1)$ is called a symmetric function if for every permutation matrix $P$,

$$
f(P \mathbf{x})=f(\mathbf{x}) \quad \text { for all } \mathbf{x} \in I^{n}
$$

Received by the editors September 8, 1995 and, in revised form, August 11, 1996.

1991 Mathematics Subject Classification. Primary 26B25, 26D05, 26D10, 52A40, 52A41, $52 \mathrm{~B} 60$.

Key words and phrases. Doubly stochastic matrix, Schur-convex function, symmetric function, isoperimetric inequality, Bonnesen inequality, degree of irregularity.

(C)1998 American Mathematical Society 
Every Schur-convex function is a symmetric function. Because if $P$ is a permutation matrix, so is its inverse $P^{-1}$. Hence if $f$ is Schur-convex, then

$$
f(\mathbf{x})=f\left(P^{-1}(P \mathbf{x})\right) \leq f(P(\mathbf{x})) \leq f(\mathbf{x}) .
$$

It shows that $f(P \mathbf{x})=f(\mathbf{x})$ for every permutation matrix $P$. On the other hand, it is not hard to see that not every symmetric function can be a Schur-convex function (cf. [15, p.258]). Similarly, every Schur-concave function is symmetric and the inverse is not true. However, we have the following so-called Schur's condition.

Lemma 1.5 ([15, p.259]). Let $f(\mathbf{x})=f\left(x_{1}, x_{2}, \cdots, x_{n}\right)$ be symmetric and have continuous partial derivatives on $I^{n}$ where $I$ is an open interval. Then $f: I^{n} \longrightarrow \mathbf{R}$ is Schur-convex if and only if

$$
\left(x_{i}-x_{j}\right)\left(\frac{\partial f}{\partial x_{i}}-\frac{\partial f}{\partial x_{j}}\right) \geq 0
$$

on $I^{n}$. It is strictly Schur-convex if (2) is a strict inequality for $x_{i} \neq x_{j}, 1 \leq i, j \leq$ $n$.

Since $f(\mathbf{x})$ is symmetric, the Schur's condition can be reduced as $([9, \mathrm{p} .57])$

$$
\left(x_{1}-x_{2}\right)\left(\frac{\partial f}{\partial x_{1}}-\frac{\partial f}{\partial x_{2}}\right) \geq 0,
$$

and $f$ is strictly Schur-convex if (3) is a strict inequality for $x_{1} \neq x_{2}$. The Schur's condition that guarantees a symmetric function being Schur-concave is the same as (2) or (3) except the direction of the inequality.

In Schur's condition, the domain of $f(\mathbf{x})$ does not have to be a Cartesian product $I^{n}$. Lemma 1.5 remains true if we replace $I^{n}$ by a set $\mathbf{A} \subset \mathbf{R}^{n}$ with the following properties ([9, p. 57]):

(i) $\mathbf{A}$ is convex and has a nonempty interior;

(ii) $\mathbf{A}$ is symmetric in the sense that $\mathbf{x} \in \mathbf{A}$ implies $P \mathbf{x} \in \mathbf{A}$ for any $n \times n$ permutation matrix $P$.

In this paper, we shall establish some analytic isoperimetric inequalities for a special class of Schur-convex functions that are made of solutions of a nonlinear second order differential equation. We will use these analytic isoperimetric inequalities to obtain some geometric inequalities. Throughout this paper, we shall be concerned with only Schur-convex functions and related inequalities. All the results will have their counterparts for Schur-concave functions.

\section{ANALYtic ISOPERIMETRIC INEQUALITIES}

In order to simplify the notations and the statements, let us set

$$
\begin{gathered}
I=(0, l) ; \quad H_{n}=\left\{\Theta=\left(\theta_{1}, \cdots, \theta_{n}\right) \in \mathbf{R}^{n} \mid \sum_{i=1}^{n} \theta_{i}=m l\right\} \quad(0<m<n) ; \\
D_{n}=I^{n} \cap H_{n} ; \quad \Omega=(\sigma, \sigma, \cdots, \sigma) \quad \text { where } \quad \sigma=\frac{1}{n} \sum_{i=1}^{n} \theta_{i}=\frac{m l}{n} .
\end{gathered}
$$

The following property of Schur-convex function plays a key role in this paper. 
Lemma 2.1. If $f: I^{n} \longrightarrow \mathbf{R}$ is a Schur-convex function, then $f(\Omega)$ is a global minimum in $D_{n}$. If $f$ is strictly Schur-convex on $I^{n}$, then $f(\Omega)$ is the unique global minimum in $D_{n}$.

Proof. Since $f$ is Schur-convex in $I^{n}$, therefore,

$$
f(S \Theta) \leq f(\Theta),
$$

for every doubly stochastic matrix $S$ and every $\Theta$ in $I^{n}$. Now, for $\Theta=\left(\theta_{1}, \cdots, \theta_{n}\right)$ in $D_{n}$, take

$$
S=\left[s_{i j}\right], \quad s_{i j}=\frac{1}{n}, \quad 1 \leq i, j \leq n .
$$

Then we have

$$
f(\Omega)=f(S \Theta) \leq f(\Theta) .
$$

If $f$ is strictly Schur-convex on $I^{n}$, then $f(\Omega)<f(\Theta)$ for all $\Theta$ in $D_{n}, \Theta \neq \Omega$.

Theorem A. Let $f(\theta)$ be a positive solution of the equation

$$
f(\theta) f^{\prime \prime}(\theta)=a_{0}+a_{1} f^{\prime}(\theta)+a_{2}\left[f^{\prime}(\theta)\right]^{2},
$$

where $a_{0}, a_{1}$ and $a_{2}$ are constants. For $\theta_{i} \in(0, l), i=1, \cdots, n ; \sum_{i=1}^{n} \theta_{i}=m l(0<$ $m<n)$; and $\sigma=\frac{m l}{n}$, suppose that $f^{\prime}(\theta) f^{\prime \prime}(\theta) \neq 0$ on $(0, l)$, and

(i) $2 f^{\prime}(\sigma)<a_{1}+\left(a_{2}+1\right)\left[f^{\prime}\left(\theta_{1}\right)+f^{\prime}\left(\theta_{2}\right)\right]$ if $f^{\prime}(\theta) f^{\prime \prime}(\theta)<0$; or

(ii) $2 f^{\prime}(\sigma)>a_{1}+\left(a_{2}+1\right)\left[f^{\prime}\left(\theta_{1}\right)+f^{\prime}\left(\theta_{2}\right)\right]$ if $f^{\prime}(\theta) f^{\prime \prime}(\theta)>0$. Then

$$
\left(\sum_{i=1}^{n} f\left(\theta_{i}\right)\right)^{2}-d_{n} \sum_{i=1}^{n} f\left(\theta_{i}\right) f^{\prime}\left(\theta_{i}\right) \geq\left[n f(\sigma)-\sum_{i=1}^{n} f\left(\theta_{i}\right)\right]^{2},
$$

where $d_{n}=n \frac{f(\sigma)}{f^{\prime}(\sigma)}$. Equality holds if and only if $\theta_{1}=\theta_{2}=\cdots=\theta_{n}=\sigma$.

Proof. Let us consider the function

$$
F(\Theta)=\left(\sum_{i=1}^{n} f\left(\theta_{i}\right)\right)^{2}-d_{n} \sum_{i=1}^{n} f\left(\theta_{i}\right) f^{\prime}\left(\theta_{i}\right)-\left[n f(\sigma)-\sum_{i=1}^{n} f\left(\theta_{i}\right)\right]^{2},
$$

and observe that $F(\Omega)=0$, where $\Omega=(\sigma, \cdots, \sigma)$. We shall prove that $F(\Theta)$ is strictly Schur-convex on $I^{n}$ where $I=(0, l)$. Then Theorem A would follow from Lemma 2.1. Since $F(\Theta)$ is obviously a symmetric function on $I^{n}$, by Lemma 1.5 and (3), to show $F(\Theta)$ is strictly Schur-convex, it suffices to verify that on $I^{n}$,

$$
\left(\theta_{1}-\theta_{2}\right)\left(\frac{\partial F}{\partial \theta_{1}}-\frac{\partial F}{\partial \theta_{2}}\right)>0, \quad \text { if } \quad \theta_{1} \neq \theta_{2} .
$$

Furthermore, let us set $L_{n}(\Theta)=\sum_{i=1}^{n} f\left(\theta_{i}\right)$. Then we have

$$
\begin{aligned}
\frac{\partial F}{\partial \theta_{i}}= & 2 L_{n}(\Theta) f^{\prime}\left(\theta_{i}\right)-d_{n}\left\{f\left(\theta_{i}\right) f^{\prime \prime}\left(\theta_{i}\right)+\left[f^{\prime}\left(\theta_{i}\right)\right]^{2}\right\} \\
& +2\left[n f(\sigma)-L_{n}(\Theta)\right] f^{\prime}\left(\theta_{i}\right) \\
= & 2 n f(\sigma) f^{\prime}\left(\theta_{i}\right)-d_{n}\left\{a_{0}+a_{1} f^{\prime}\left(\theta_{i}\right)+\left(a_{2}+1\right)\left[f^{\prime}\left(\theta_{i}\right]^{2}\right\}, \quad i=1,2 .\right. \\
\frac{\partial F}{\partial \theta_{1}}-\frac{\partial F}{\partial \theta_{2}}= & 2 n f(\sigma)\left[f^{\prime}\left(\theta_{1}\right)-f^{\prime}\left(\theta_{2}\right)\right] \\
& -d_{n}\left\{a_{1}\left[f^{\prime}\left(\theta_{1}\right)-f^{\prime}\left(\theta_{2}\right)\right]+\left(a_{2}+1\right)\left[\left(f^{\prime}\left(\theta_{1}\right)\right)^{2}-\left(f^{\prime}\left(\theta_{2}\right)\right)^{2}\right]\right\} \\
= & 2 d_{n}\left[f^{\prime}\left(\theta_{1}\right)-f^{\prime}\left(\theta_{2}\right)\right]\left\{f^{\prime}(\sigma)-\left[\frac{a_{1}}{2}+\frac{a_{2}+1}{2}\left(f^{\prime}\left(\theta_{1}\right)+f^{\prime}\left(\theta_{2}\right)\right)\right]\right\} .
\end{aligned}
$$


Therefore,

$$
\begin{aligned}
& \left(\theta_{1}-\theta_{2}\right)\left(\frac{\partial F}{\partial \theta_{1}}-\frac{\partial F}{\partial \theta_{2}}\right) \\
& \quad=2 d_{n}\left(\theta_{1}-\theta_{2}\right)\left[f^{\prime}\left(\theta_{1}\right)-f^{\prime}\left(\theta_{2}\right)\right]\left\{f^{\prime}(\sigma)-\frac{a_{1}}{2}-\frac{a_{2}+1}{2}\left[f^{\prime}\left(\theta_{1}\right)+f^{\prime}\left(\theta_{2}\right)\right]\right\} .
\end{aligned}
$$

Case (i). $f^{\prime}(\theta) f^{\prime \prime}(\theta)<0$.

If $f^{\prime \prime}(\theta)<0,\left(f^{\prime}(\theta)>0\right)$, then $f^{\prime}$ is decreasing on $(0, l)$ and $d_{n}>0$; if $f^{\prime \prime}(\theta)>0$, $\left(f^{\prime}(\theta)<0\right)$, then $f^{\prime}$ is increasing on $(0, l)$ and $d_{n}<0$; so if $\theta_{1} \neq \theta_{2}$, we always have

$$
2 d_{n}\left(\theta_{1}-\theta_{2}\right)\left[f^{\prime}\left(\theta_{1}\right)-f^{\prime}\left(\theta_{2}\right)\right]<0 .
$$

Moreover, by the hypothesis (i),

$$
f^{\prime}(\sigma)<\frac{a_{1}}{2}+\frac{a_{2}+1}{2}\left[f^{\prime}\left(\theta_{1}\right)+f^{\prime}\left(\theta_{2}\right)\right] .
$$

Thus we have

$$
\left(\theta_{1}-\theta_{2}\right)\left(\frac{\partial F}{\partial \theta_{1}}-\frac{\partial F}{\partial \theta_{2}}\right)>0, \quad \text { for } \quad \theta_{1} \neq \theta_{2} .
$$

Case (ii). $f^{\prime}(\theta) f^{\prime \prime}(\theta)>0$.

If $f^{\prime \prime}(\theta)>0,\left(f^{\prime}(\theta)>0\right)$, then $f^{\prime}$ is increasing on $(0, l)$ and $d_{n}>0$; if $f^{\prime \prime}(\theta)<0$, $\left(f^{\prime}(\theta)<0\right)$, then $f^{\prime}$ is decreasing on $(0, l)$ and $d_{n}<0$; so if $\theta_{1} \neq \theta_{2}$, we always have

$$
2 d_{n}\left(\theta_{1}-\theta_{2}\right)\left[f^{\prime}\left(\theta_{1}\right)-f^{\prime}\left(\theta_{2}\right)\right]>0 .
$$

By hypothesis (ii),

$$
f^{\prime}(\sigma)>\frac{a_{1}}{2}+\frac{a_{2}+1}{2}\left[f^{\prime}\left(\theta_{1}\right)+f^{\prime}\left(\theta_{2}\right)\right] .
$$

We still have

$$
\left(\theta_{1}-\theta_{2}\right)\left(\frac{\partial F}{\partial \theta_{1}}-\frac{\partial F}{\partial \theta_{2}}\right)>0, \quad \text { for } \quad \theta_{1} \neq \theta_{2}
$$

Corollary 2.2. Let $\theta_{i} \in(0, \pi / 2), i=1,2, \cdots, n$; and $\sum_{i=1}^{n} \theta_{i}=\pi$. Then

$$
\left(\sum_{i=1}^{n} \sin \theta_{i}\right)^{2}-d_{n} \sum_{i=1}^{n} \sin \theta_{i} \cos \theta_{i} \geq\left[n \sin \frac{\pi}{n}-\sum_{i=1}^{n} \sin \theta_{i}\right]^{2},
$$

where $d_{n}=n \tan \frac{\pi}{n}$. Equality holds if and only if $\theta_{1}=\theta_{2}=\cdots=\theta_{n}=\frac{\pi}{n}$.

Proof. In equation (4), let $a_{0}=-1, a_{1}=0$ and $a_{2}=1$. Then $f(\theta)=\sin \theta$ is a positive solution of (4), and

$$
f^{\prime}(\theta) f^{\prime \prime}(\theta)=-\sin \theta \cos \theta<0, \quad \text { for } \quad \theta \in(0, \pi / 2) .
$$

Without loss of generality, assume that $\theta_{1} \leq \theta_{2} \leq \cdots \leq \theta_{n}$, then we have

$$
f^{\prime}(\sigma)=\cos \frac{\pi}{n}<\cos \theta_{1}+\cos \theta_{2}=\frac{a_{1}}{2}+\frac{a_{2}+1}{2}\left[f^{\prime}\left(\theta_{1}\right)+f^{\prime}\left(\theta_{2}\right)\right] .
$$

Therefore the corollary follows as a special case of Theorem A (i). 
Similarly, we also have a special case of Theorem A (ii).

Corollary 2.3. Let $\theta_{i} \in(0, \pi / 2), i=1,2, \cdots, n$; and $\sum_{i=1}^{n} \theta_{i}=\pi$. Then

$$
\left(\sum_{i=1}^{n} \cos \theta_{i}\right)^{2}-d_{n} \sum_{i=1}^{n} \sin \theta_{i} \cos \theta_{i} \geq\left[n \cos \frac{\pi}{n}-\sum_{i=1}^{n} \cos \theta_{i}\right]^{2},
$$

where $d_{n}=n \cot \frac{\pi}{n}$. Equality holds if and only if $\theta_{1}=\theta_{2}=\cdots=\theta_{n}=\frac{\pi}{n}$.

Remark. The solutions of equation (4) includes many important functions in addition to sine and cosine functions. We could establish more analytic isoperimetric inequalities by using different solutions of (4). As we shall see in the next section, many special cases of (5) yield interesting geometric inequalities. Even within the scope of analytic inequalities, Theorem A has many by-products. The following one is an example for concave functions.

Corollary 2.4. Let $f(\theta)$ be a positive solution of the equation

$$
f(\theta) f^{\prime \prime}(\theta)=a_{0}+a_{1} f^{\prime}(\theta)+a_{2}\left[f^{\prime}(\theta)\right]^{2}
$$

on $(0, l)$, where $a_{0}, a_{1}$ and $a_{2}$ are constants; for $\theta_{i} \in(0, l), i=1, \cdots, n ; \sum_{i=1}^{n} \theta_{i}=$ $m l(0<m<n)$; and $\sigma=\frac{m l}{n}$. Suppose that $f^{\prime \prime}(\theta)<0$ and

(i) $2 f^{\prime}(\sigma)<a_{1}+\left(a_{2}+1\right)\left[f^{\prime}\left(\theta_{1}\right)+f^{\prime}\left(\theta_{2}\right)\right]$ if $f^{\prime}(\theta)>0$;

(ii) $2 f^{\prime}(\sigma)>a_{1}+\left(a_{2}+1\right)\left[f^{\prime}\left(\theta_{1}\right)+f^{\prime}\left(\theta_{2}\right)\right]$ if $f^{\prime}(\theta)<0$. Then

$$
2 \sum_{i=1}^{n} f\left(\theta_{i}\right) \geq \sum_{i=1}^{n} f\left(\theta_{i}\right) W_{i}+n f(\sigma)
$$

where $W_{i}=\frac{f^{\prime}\left(\theta_{i}\right)}{f^{\prime}(\sigma)}, i=1, \cdots, n$. Equality holds if and only if $\theta_{1}=\theta_{2}=\cdots=\theta_{n}$.

Proof. From the inequality (5) in Theorem A, we see that

$$
-d_{n} \sum_{i=1}^{n} f\left(\theta_{i}\right) f^{\prime}\left(\theta_{i}\right) \geq n^{2} f^{2}(\sigma)-2 n f(\sigma) \sum_{i=1}^{n} f\left(\theta_{i}\right),
$$

that is,

$$
\begin{aligned}
2 \sum_{i=1}^{n} f\left(\theta_{i}\right) & \geq \frac{1}{f^{\prime}(\sigma)} \sum_{i=1}^{n} f\left(\theta_{i}\right) f^{\prime}\left(\theta_{i}\right)+n f(\sigma) \\
& =\sum_{i=1}^{n} f\left(\theta_{i}\right) W_{i}+n f(\sigma) . \quad \square
\end{aligned}
$$

Note. For a concave function $f(\theta)\left(f^{\prime \prime}(\theta)<0\right)$, the following Jensen's inequality is well-known $([3],[10])$ :

$$
\sum_{i=1}^{n} f\left(\theta_{i}\right) \leq n f(\sigma)
$$

where equality holds if and only if $\theta_{1}=\cdots=\theta_{n}=\sigma$.

Corollary 2.4 says that if $f(\theta)$ and its derivatives are subject to certain differential equation and differential inequalities, we may have an inequality that is different from (9). Comparing (8) and (9), it is interesting to note that the hypotheses in 
the Corollary 2.4 are sufficient conditions for the following "weighted inequality":

$$
\sum_{i=1}^{n} f\left(\theta_{i}\right) \geq \sum_{i=1}^{n} f\left(\theta_{i}\right) W_{i}
$$

where $W_{i}=\frac{f^{\prime}\left(\theta_{i}\right)}{f^{\prime}(\sigma)}>0, i=1, \cdots, n$. Equality holds if and only if $\theta_{1}=\cdots=\theta_{n}=\sigma$.

\section{GEOMETRIC ISOPERIMETRIC INEQUALITIES}

In this section, we apply those analytic isoperimetric inequalities obtained in section 2 to provide simple proofs for some geometric inequalities which are, if not completely new, at least not easy to find in the literature of elementary geometry.

Let us recall the classical isoperimetric inequality for an $n$-sided plane polygon $P_{n}$, that is,

$$
L_{n}^{2}-4 d_{n} A_{n} \geq 0,
$$

where $L_{n}$ is the perimeter of $P_{n}, A_{n}$ is the area of the domain enclosed by $P_{n}$, and $d_{n}=n \tan \frac{\pi}{n}$. Equality holds if and only if $P_{n}$ is regular ([4], [5], [7], [11]).

In geometry, we call $L_{n}^{2}-4 d_{n} A_{n}$ the isoperimetric deficit of the polygon $P_{n}$. It measures the deviation of $P_{n}$ from the "regularity". Inequality (10) can be improved to the following so-called Bonnesen-style isoperimetric inequality ([12], [17]):

$$
L_{n}^{2}-4 d_{n} A_{n} \geq B_{n}, \quad d_{n}=n \tan \frac{\pi}{n},
$$

where the quantity $B_{n}$ satisfies (i) $B_{n} \geq 0$; (ii) $B_{n}=0$ only when $P_{n}$ is regular; (iii) $B_{n}$ has geometric significance. It is important to find various $B_{n}$ 's, since they will provide lower bounds for the isoperimetric deficit of $P_{n}$. For a comprehensive discussion on Bonnesen-style inequalities, refer to [12], [17].

From plane geometry, it is known that among all $n$-sided plane polygons with given $n$ sides, the one which can be inscribed in a circle encloses the largest area ([4], $[7])$. A polygon is called "cyclic" if it can be inscribed in a circle. To investigate isoperimetric inequalities for plane polygons, we need to pay attention to cyclic polygons only. The following Bonnesen-style isoperimetric inequality for cyclic polygons is a direct consequence of Corollary 2.2.

Theorem 3.1 ([17]). Let $P_{n}$ be an n-sided plane polygon inscribed in a circle of radius $R$ with perimeter $L_{n}$, enclosing a domain of area $A_{n}$. Then

$$
L_{n}^{2}-4 d_{n} A_{n} \geq\left(l_{n}-L_{n}\right)^{2}, \quad d_{n}=n \tan \frac{\pi}{n},
$$

where $l_{n}$ is the perimeter of the regular $n$-sided polygon inscribed in the same circle with $P_{n}$. Equality holds if and only if $P_{n}$ is also regular.

Proof. If $a_{i}$ denotes the length of the $i$ th side of $P_{n}$, and $\theta_{i}$ denotes half of the central angle subtended by the $i$ th side of $P_{n}, i=1, \cdots, n$, then

$$
\begin{array}{cc}
L_{n}=\sum_{i=1}^{n} a_{i}= & \sum_{i=1}^{n} 2 R \sin \theta_{i} ; \quad A_{n}=\sum_{i=1}^{n} \frac{1}{2} a_{i} R \cos \theta_{i}=\sum_{i=1}^{n} R^{2} \sin \theta_{i} \cos \theta_{i} ; \\
\sum_{i=1}^{n} \theta_{i}=\pi ; & l_{n}=2 n R \sin \frac{\pi}{n} .
\end{array}
$$

Substitute the information above into (12), then Theorem 3.1 follows from Corollary 2.2 . 
Similarly, if we define the $i$ th altitude $h_{i}$ of $P_{n}$ as the distance from the centroid of $P_{n}$ to the $i$ th side of $P_{n}, i=1,2, \cdots, n$, and the total altitude of $P_{n}$ as $H_{n}=$ $\sum_{i=1}^{n} h_{i}$, then we have the following geometric interpretation of Corollary 2.3. The proof shall be omitted since it is almost identical with the proof of Theorem 3.1.

Theorem 3.2. Let $P_{n}$ be an n-sided plane polygon inscribed in a circle of radius $R$ with total altitude $H_{n}$, enclosing a domain of area $A_{n}$. Then

$$
H_{n}^{2}-d_{n} A_{n} \geq\left(\bar{H}_{n}-H_{n}\right)^{2}, \quad d_{n}=n \cot \frac{\pi}{n},
$$

where $\bar{H}_{n}=n R \cos \frac{\pi}{n}$ is the total altitude of the regular $n$-sided polygon inscribed in the same circle with $P_{n}$. Equality holds if and only if $P_{n}$ is also regular.

Now, for a cyclic polygon $P_{n}$ with lengths of sides $a_{1}, a_{2}, \cdots, a_{n}$, if $2 \theta_{1}, 2 \theta_{2}, \cdots$, $2 \theta_{n}$ are the central angles subtended by the $n$ sides respectively, we shall denote $P_{n}$ by $P_{n}(\Theta)$ where $\Theta=\left(\theta_{1}, \theta_{2}, \cdots, \theta_{n}\right), \sum_{i=1}^{n} \theta_{i}=\pi$, and introduce the degree of irregularity for $P_{n}(\Theta)$.

Definition 3.3. Let $P_{n}(\Theta)$ be an $n$-sided cyclic polygon. The degree of irregularity of $P_{n}(\Theta)$ is defined as

$$
\operatorname{Deg}\left[P_{n}(\Theta)\right]=\left(\sum_{i=1}^{n} \sin \theta_{i}\right)^{2}-d_{n} \sum_{i=1}^{n} \sin \theta_{i} \cos \theta_{i}-\left[n \sin \frac{\pi}{n}-\sum_{i=1}^{n} \sin \theta_{i}\right]^{2},
$$

where $d_{n}=n \tan \frac{\pi}{n}$.

It is clear that $\operatorname{Deg}\left[P_{n}(\Theta)\right] \geq 0$ for all $P_{n}(\Theta)$, and $\operatorname{Deg}\left[P_{n}(\Theta)\right]=0$ only when $P_{n}(\Theta)$ is regular. We have noticed that the equality in (10) or (11) distinguishes only the regular polygon among all isoperimetric polygons with the same number of sides. Definition 3.3 allows us to compare any two $n$-sided cyclic polygons in terms of their degrees of irregularity.

Theorem 3.4. Let $P_{n}(\Theta)$ and $Q_{n}(\bar{\Theta})$ be two n-sided cyclic polygons. If there is a doubly stochastic matrix $S$ such that $\bar{\Theta}=S \Theta$, then

$$
\operatorname{Deg}\left[Q_{n}(\bar{\Theta})\right] \leq \operatorname{Deg}\left[P_{n}(\Theta)\right] .
$$

Equality holds if and only if $\Theta$ and $\bar{\Theta}$ differ by a permutation.

Proof. Let $F(\Theta)=\operatorname{Deg}\left[P_{n}(\Theta)\right]$; then $F(\bar{\Theta})=\operatorname{Deg}\left[Q_{n}(\bar{\Theta})\right]$. From Corollary 2.2 we see that $F$ is Schur-convex, and hence

$$
F(\bar{\Theta})=F(S \Theta) \leq F(\Theta) .
$$

Remark. $\operatorname{Deg}\left[P_{n}(\Theta)\right]$ is a homothetic invariant for cyclic polygons. Hence if $P_{n}(\Theta)$ and $Q_{n}(\bar{\Theta})$ are two $n$-sided homothetic cyclic polygons, that is, $\Theta=\bar{\Theta}$ (they have the same shape but may have different size), then they have the same degree of irregularity. On the other hand, $\operatorname{Deg}\left[P_{n}(\Theta)\right]=\operatorname{Deg}\left[Q_{n}(\bar{\Theta})\right]$ does not necessarily imply the homotheticity of $P_{n}(\Theta)$ and $Q_{n}(\bar{\Theta})$, because $\Theta$ and $\bar{\Theta}$ may differ by a permutation so that the two cyclic polygons may not be obtained from each other by a homothetic transformation. If we consider the class of all $n$-sided cyclic polygons $(n>3)$, the degree of irregularity can be used to classify the polygons according to their deviations from the regularity. 
Let us consider an $n$-sided plane polygon $P_{n}$ that circumscribes the unit circle with points of tangency $A_{1}, A_{2}, \cdots, A_{n}$, and denote by $2 \theta_{1}, \cdots, 2 \theta_{n}$ the $n$ central angles in the unit circle subtended by the $n$ arcs $\widehat{A_{1} A_{2}}, \cdots, \widehat{A_{n} A_{n+1}}\left(A_{n+1}=A_{1}\right)$ respectively. Once again, denote the polygon as $P_{n}(\Theta)$, where $\Theta=\left(\theta_{1}, \cdots, \theta_{n}\right)$, and $\sum_{i=1}^{n} \theta_{i}=\pi$. Consider the probability measures $\mu$ that are circularly symmetric, i.e., measures that are invariant under rotations about the origin. Write $\mu\left(P_{n}(\Theta)\right)$ to denote the probability content of the polygon $P_{n}(\Theta)$, Wynn had proved the following result in 1977 ([9], [16]).

Theorem 3.5 ([9, p.213]). If $P_{n}(\Theta)$ and $Q_{n}(\bar{\Theta})$ are two n-sided plane polygons that circumscribe the unit circle, then for every spherically symmetric probability measure $\mu$,

$$
\mu\left(P_{n}(\Theta)\right) \geq \mu\left(Q_{n}(\bar{\Theta})\right) \quad \text { if and only if } \quad \bar{\Theta}=S \Theta
$$

for some doubly stochastic matrix $S$.

For a cyclic plane polygon $P_{n}$, the degree of irregularity of $P_{n}$ is obviously associated with a spherically symmetric probability measure. Then, Theorem 3.4 might be viewed as a dual result to Theorem 3.5. More generally, the definition of $\operatorname{Deg}\left[P_{n}(\Theta)\right]$ could be introduced to the polygonal curves in the surfaces of constant curvature (cf. [6]). For a different purpose, one may use (7) to define the degree of irregularity of a cyclic polygon that would also describe the deviation of the polygon from regularity. It would also be another homothetic invariant for cyclic polygons.

To conclude this section, it is worth pointing out a geometric interpretation of Corollary 2.4. If we take $f(\theta)=\sin \theta$, which is a concave function on $\left(0, \frac{\pi}{2}\right)$, then a special case of $(8)$ is

$$
2 \sum_{i=1}^{n} \sin \theta_{i} \geq \sec \frac{\pi}{n} \sum_{i=1}^{n} \sin \theta_{i} \cos \theta_{i}+n \sin \frac{\pi}{n}
$$

with equality if and only if $\theta_{1}=\theta_{2}=\cdots=\theta_{n}=\frac{\pi}{n}$.

Multiplying a positive number $R^{2}$ by each term above yields the following notable isoperimetric inequality for a cyclic polygon.

Theorem 3.6. Let $P_{n}$ be an $n$-sided polygon inscribed in a circle of radius $R$. If $P_{n}$ has a perimeter $L_{n}$ and encloses a domain of area $A_{n}$, then

$$
R L_{n} \geq c_{n} A_{n}+\frac{1}{c_{n}} d_{n} R^{2},
$$

where $c_{n}=\sec \frac{\pi}{n}, d_{n}=n \tan \frac{\pi}{n}$, and equality holds if and only if $P_{n}$ is regular.

Let us recall that if $C$ is a simple closed plane curve with perimeter $L$, and enclosing a domain of area $A$, then $([12, \mathrm{p} .3])$,

$$
t L \geq A+\pi t^{2},
$$

for all $t$ such that $\rho \leq t \leq R$, where $\rho$ and $R$ are the inradius and circumradius of $C$ respectively. This inequality is the most important one in [12] and produces many equivalent Bonnesen-style inequalities for $C$. If we consider an $n$-sided plane polygon $P_{n}$ with inradius $\rho$, it is also well-known that $([11, \mathrm{p} .1210])$,

$$
\rho L_{n} \geq A_{n}+d_{n} \rho^{2}, \quad d_{n}=n \tan \frac{\pi}{n} .
$$


We wish to establish a general inequality similar to (17) for a polygon $P_{n}$ as follows:

$$
t L_{n} \geq A_{n}+d_{n} t^{2}, \quad d_{n}=n \tan \frac{\pi}{n} .
$$

Note. This would be a stronger inequality than (17) for a polygon $P_{n}$ since $d_{n}>$ $\pi$ for $n>3$. From (18), we find that the parameter $t$ in (19) can be as small as the inradius $\rho$ of $P_{n}$. However, from (16) we find it cannot be as large as the circumradius $R$ of $P_{n}$. Comparison of (16), (18) and (17) demonstrates some differences between the Bonnesen-style inequalities for smooth plane curves and the Bonnesen-style inequalities for plane polygonal curves.

\section{Concluding Remarks}

The results in sections 2 and 3 suggest that a special class of Schur-convex functions would imply some interesting analytic and geometric isoperimetric inequalities if they are made from the positive solutions of the equation

$$
y y^{\prime \prime}=a_{0}+a_{1} y^{\prime}+a_{2}\left[y^{\prime}\right]^{2},
$$

and satisfy the appropriate first order differential inequality. Observe that (20) is a nonlinear second order differential equation with the variable $x$ missing. By a standard technique taught in undergraduate differential equation courses (cf. [2]), set $v=y^{\prime}$, then $y^{\prime \prime}=v \frac{d v}{d y}$. The equation (20) can be written as

$$
\frac{v}{a_{0}+a_{1} v+a_{2} v^{2}} d v=\frac{1}{y} d y .
$$

One may obtain many important solutions of this differential equation for different choices on the coefficients $a_{0}, a_{1}$, and $a_{2}$. For instance,

1) $y=\sin x, \cos x$, and $\sinh x$

2) $y=\cosh x$

3) $y=\tan x, \tanh x, \operatorname{coth} x$

4) $y=\cot x$

$$
\begin{gathered}
\left(a_{0}=-1, a_{1}=0, a_{2}=1\right) ; \\
\left(a_{0}=1, a_{1}=0, a_{2}=1\right) ; \\
\left(a_{0}=0, a_{1}=-2, a_{2}=2\right) ; \\
\left(a_{0}=0, a_{1}=2, a_{2}=2\right) ;
\end{gathered}
$$

5) $y=x^{\alpha}, \alpha$ is a constant $\quad\left(a_{0}=0, a_{1}=0, a_{2}=\frac{\alpha-1}{\alpha}\right)$;

6) $y=A e^{\alpha x}+B e^{-\alpha x}, \alpha, A>0$, and $B>0$ are constants $\left(a_{0}=4 A B \alpha^{2}, a_{1}=0\right.$, $\left.a_{2}=1\right)$.

In [5], [6], [17] and [18], we have proved many discrete analytic isoperimetric inequalities for a large class of special functions. It turns out that most of them can be derived from inequalities that are associated with Schur-convex functions or Schur-concave functions. As we have learned in [9, ch.8] and as the results have illustrated in this paper, Schur-convexity seems to be a nice and simple idea in the study of geometric isoperimetric inequalities on surfaces of constant curvature.

\section{REFERENCES}

1. E. B. Beckenbach and R. Bellman, Inequalities, 2nd ed., Springer-Verlag, Berlin and New York, 1965. MR 33:236

2. William E. Boyce and Richard C. DiPrima, Elementary Differential Equations and Boundary Value Problems, 5th Ed., John Wiley \& Sons, Inc., New York·Chichester-Brisbane-Toronto -Singapore, 1992. MR 31:3651

3. G. Hardy, J. E. Littlewood and G. Polya, Inequalities, Cambridge Univ. Press, Cambridge, New York, 1951. MR 13:727e

4. N. D. Kazarinoff, Geometric Inequalities, New Math. Library, Math. Assoc. of America, 1961. MR 24:A1 
5. H. T. Ku, M. C. Ku and X. M. Zhang, Analytic and Geometric Isoperimetric Inequalities, Journal of Geometry, vol.53(1995)100-121. MR 96f:52012

6. H. T. Ku, M. C. Ku and X. M. Zhang, Isoperimetric Inequalities on the Surfaces of Constant Curvature, (Preprint).

7. D. S. Macnab, Cyclic Polygons and Related Questions, Math. Gazette, 65(1981)22-28. MR 82j:51029

8. J. E. Marsden and A. J. Tromba, Vector Calculus, 2nd Ed., W. H. Freeman and Company, 1981.

9. Albert W. Marshall and Ingram Olkin, Inequalities: Theory of Majorization and Its Applications, Academic Press, 1979. MR 81b:00002

10. D. S. Mitrinović, Analytic Inequalities, Springer-Verlag, Berlin, Heidelberg, New York, 1970. MR 43:448

11. R. Osserman, The isoperimetric inequality, Bull. Amer. Math. Soc. 84(1978), 1182-1238. MR 58:18161

12. R.Osserman, Bonnesen-style Isoperimetric Inequalities, Amer.Math.Monthly, 86 (1979)1-29. MR 80h:52013

13. A. Ostrowski, Sur quelques application des fonctions convexes et concave au sens de I. Schur, J. Math. Pures Appl. 31(1952) 253-292. MR 14:625d

14. I. Schur, Über eine Klasse von Mittelbildungen mit Anwendungen auf die Determinantentheorie, Sitzunsber. Berlin. Math. Ges. 22(1923)9-20.

15. A. Wayne Roberts and Dale E. Varberg, Convex Functions, Academic Press, New York, San Francisco, London, 1973. MR 56:1201

16. H.Wynn, An inequality for certain bivariate probability integrals, Biometrika, 64(1977)411414. MR 58:18892

17. X.M.Zhang, Bonnesen-style Inequalities and Pseudo-perimeters for Polygons, Journal of Geometry, to appear.

18. X.M.Zhang, A Refinement of the Discrete Wirtinger Inequality, Journal of Mathematical Analysis and Applications, 200(1996)687-697. MR 97h:26012

Department of Mathematics and Statistics, University of South Alabama, Mobile, Alabama 36688

E-mail address: zhang@mathstat.usouthal.edu 